TOKYO J. MATH.

VOL. 28, No. 1, 2005

\title{
On the Density Function of an Invariant Measure under One-Dimensional Bernoulli Transformations
}

\author{
Yuko ICHIKAWA
}

Tokyo National College of Technology

(Communicated by Y. Maeda)

\begin{abstract}
The continuity of the density function of the invariant probability measure for piecewise $C^{2}$ Bernoulli transformations is proved.

\section{Introduction}

The purpose of this article is to show that the continuity of the density function of an invariant probability measure for piecewise $C^{2}$, expanding, and Bernoulli transformations of the unit interval $[0,1]$. We study Markov (not necessarily Bernoulli) transformations approximating them by piecewise linear transformations. To deal piecewise linear transformations as symbolic dynamics, Mori defined Fredholm matrices in [2] and [3]. First using these matrices, we construct a recurrent formula between the eigenvectors of them, and show the existence of an eigenfunction of Perron-Frobenius operator of original transformation which is the density function of the invariant probability measure. Secondly, we show that the density function is continuous for Bernoulli transformations. For piecewise $C^{2}$ and expanding transformation, Lasota and York have shown the existence of the invariant measure in [1]. The first part of this paper gives another proof of the Lasota and York's result for restricted cases.
\end{abstract}

\section{Notations and Results}

Let $F:[0,1] \rightarrow[0,1]$ satisfy the following conditions.

(C1): piecewise $C^{2}$. There exists a partition $0=p_{0}<p_{1}<\cdots<p_{r}=1$ of $[0,1]$ such that the restriction of $F$ to $\left(p_{i-1}, p_{i}\right), i=1,2, \cdots, r$ is $C^{2}$ and monotone function which can be extended to $\left[p_{i-1}, p_{i}\right]$ as a $C^{2}$ function. We call a set $\mathcal{A}=\left\{a_{1}, a_{2}, \cdots, a_{r}\right\}$ 'alphabet' and $\left(p_{i-1}, p_{i}\right)$ is labeled by $\left\langle a_{i}\right\rangle$. Here $\# \mathcal{A}=r$ is finite.

Received August 16, 2002; revised February 21, 2005 
(C2): transitive. For any $x, y \in[0,1]$, and for any neighborhoods $V(x), V(y)$ of $x$ and $y$, respectively, there exists $n \in \mathbf{N}$ such that

$$
F^{n}(V(x)) \cap V(y) \neq \emptyset,
$$

here $F^{n}$ is the $n$-th iteration of $F$.

\section{(C3): expanding.}

$$
\xi \equiv \liminf _{n \rightarrow \infty} \underset{x \in[0,1]}{\operatorname{essinf}} \frac{1}{n} \log \left|F^{n^{\prime}}(x)\right|>0 .
$$

$F$ is called 'Markov' if for any $a \in \mathcal{A}$, there exist letters $b_{1}, b_{2}, \cdots, b_{k}\left(b_{i} \in \mathcal{A}\right)$ such that

$$
\overline{F(\langle a\rangle)}=\bigcup_{i=1}^{k} \overline{\left\langle b_{i}\right\rangle},
$$

where $\bar{J}$ stands for the closure of $J . F$ is called 'Bernoulli', if $F$ for any $a \in \mathcal{A}, \overline{F(\langle a\rangle)}=$ $[0,1]$. Throughout this paper, we assume that $F$ is Markov, and in section 5 we assume that $F$ is Bernoulli.

To express $F$ as symbolic dynamics, we prepare several notation. We call a finite sequence of letters $w=b_{1} b_{2} \cdots b_{n}\left(b_{k} \in \mathcal{A}\right)$ a word, and we define

$$
\begin{aligned}
& |w|=n \quad \text { (the length of a word), } \\
& w[k]=b_{k} \quad \text { for } 1 \leq k \leq|w| \quad(n \text {-th coordinate) } \\
& w[k, l]=b_{k} b_{k+1} \cdots b_{l} \quad \text { for } 1 \leq k<l \leq|w| \\
& \langle w\rangle=\bigcap_{i=1}^{n} F^{-i+1}(\langle w[i]\rangle), \\
& h(w)=b_{1} \cdots b_{n-1} \\
& t(w)=b_{2} \cdots b_{n} .
\end{aligned}
$$

We say a word $w F$-admissible if $\langle w\rangle \neq \emptyset$, and define the sets of $F$-admissible words as follows:

$$
\begin{aligned}
& W_{n}=\left\{w \in \mathcal{A}^{n}:|w|=n, w \text { is } F \text {-admissible }\right\} \\
& \tilde{W}_{n}=\bigcup_{k=0}^{n} W_{k}=\{w: w \text { is } F \text {-admissible, }|w| \leq n\}, \\
& W_{\infty}=\left\{w \in \mathcal{A}^{\mathbf{N}}: w[1, n] \in W_{n} \text { for all } n\right\} .
\end{aligned}
$$

It is well known that there exists a unique invariant probability measure $\mu$ under $F$ and the dynamical system $([0,1], \mu, F)$ is mixing, therefore it is ergodic. From the condition that $F$ is expanding, for any $\varepsilon>0$ there exists $N_{0}$ such that for any $N \geq N_{0}$ and for any $w \in W_{N}$,

$$
\operatorname{Lebes}(\langle w\rangle) \leq e^{-(\xi-\varepsilon) N}
$$

where Lebes $(\langle w\rangle)$ denote the Lebesgue measure of $\langle w\rangle$.

Let us introduce orders among admissible words. 
Definition 1. For two $F$-admissible words $w$ and $w^{\prime}$, we define $w<w^{\prime}$ if one of the following holds:

1. $|w|<\left|w^{\prime}\right|$

2. $|w|=\left|w^{\prime}\right|$ and $x<y$ holds for all $x \in\langle w\rangle$ and $y \in\left\langle w^{\prime}\right\rangle$.

The orders in $W_{N}$ and $W_{\infty}$ are introduced by the above definition.

Let $\mathcal{P}_{N}=\left\{\langle w\rangle: w \in W_{N}\right\}$. Then $\mathcal{P}_{N}$ gives a partition of [0,1]. For $M<N, \mathcal{P}_{N}$ is a refinement of $\mathcal{P}_{M}$. On $\langle w\rangle \in \mathcal{P}_{N}$, we define a piecewise linear transformation $F_{N}$, whose graph is the segment from $\left(p_{w}^{-}, \lim _{x \downarrow p_{w}^{-}} F(x)\right)$ to $\left(p_{w}^{+}, \lim _{x \uparrow p_{w}^{+}} F(x)\right)$, where $p_{w}^{-}$and $p_{w}^{+}$are the left and the right end points of $\langle w\rangle$, respectively. We call $F_{N}$ the $N$-th approximation of $F$. Let $\eta_{w}=\left|\left(F_{N} \mid\langle w\rangle\right)^{\prime}\right|^{-1}$. Here, we note that for $w \in W_{N}, \bigcap_{i=1}^{|w|} F_{N}^{-i+1}(\langle w[i]\rangle)=\langle w\rangle$. That is, for $w \in \tilde{W}_{N},\langle w\rangle$ is equal under $F$ and $F_{N}$.

Let $P: L^{1} \rightarrow L^{1}$ be the Perron-Frobenius operator associated with $F$, that is, for $f \in L^{1}$,

$$
P f(x)=\sum_{y: F(y)=x} f(y)\left|F^{\prime}(y)\right|^{-1},
$$

and $P_{N}$ be the one associated with $F_{N}$.

Operating $P_{1}$ to the indicator function $1_{\langle a\rangle}(a \in \mathcal{A})$, from the Markov condition (1) we obtain

$$
P_{1} 1_{\langle a\rangle}(x)=\eta_{a} \sum_{b: a b \in W_{2}} 1_{\langle b\rangle}(x)
$$

In general, for $w \in W_{N}$,

$$
P_{N} 1_{\langle w\rangle}(x)=\eta_{w} \sum_{b: w b \in W_{N+1}} 1_{\langle t(w b)\rangle}(x) .
$$

Let $\Phi_{N}$ be the Fredholm matrix for $F_{N}$, that is, $\Phi_{N}$ is a $W_{N} \times W_{N}$ matrix:

$$
\left(\Phi_{N}\right)_{w, w^{\prime}}= \begin{cases}\eta_{w} & t(w)=h\left(w^{\prime}\right), \\ 0 & \text { otherwise }\end{cases}
$$

For a partition $\mathcal{P}_{N}$, let $\boldsymbol{i}_{N}$ and $|\boldsymbol{i}|_{N}$ be the vectors corresponding to words $w \in W_{N}$, whose components are $\left(\boldsymbol{i}_{N}\right)_{w}=1_{\langle w\rangle}$, and $\left(|\boldsymbol{i}|_{N}\right)_{w}=\operatorname{Lebes}(\langle w\rangle)$, respectively. Then the equations (3) can be written

$$
P_{N} 1_{\langle w\rangle}=\left(\Phi_{N} \boldsymbol{i}_{N}\right)_{w}
$$

EXAMPLE 1. Let

$$
F(x)= \begin{cases}x / \eta_{a} & 0 \leq x \leq \eta_{a} \\ \left(x-\eta_{a}\right) / \eta_{b} & \eta_{a} \leq x \leq 1\end{cases}
$$



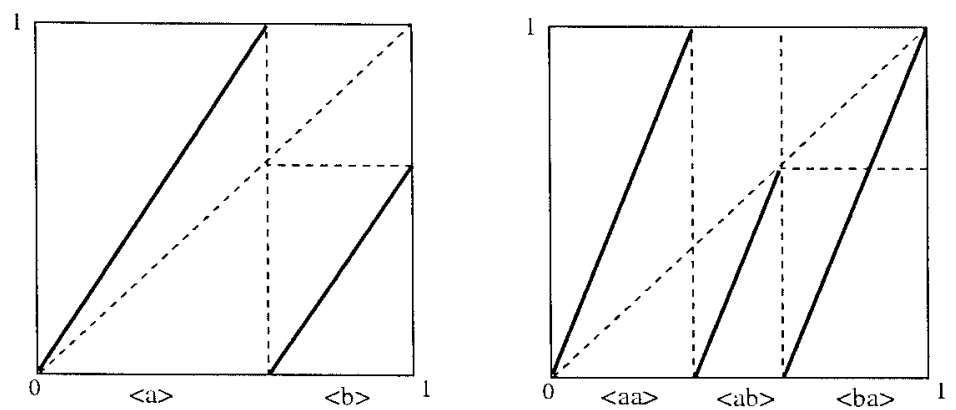

FIGURE 1. $\quad F(x)$ and $F^{2}(x)$

Here, $\frac{1}{2}<\eta_{a}<1$ and $1-\eta_{a}=\eta_{a} \eta_{b}$ holds. Fig. 1 shows the graphs of $F(x)$ and $F^{2}(x)$. Then $\mathcal{A}=\{a, b\}$, and $\langle a\rangle=\left(0, \eta_{a}\right),\langle b\rangle=\left(\eta_{a}, 1\right) . W_{2}=\{a a, a b, b a\}$. For this transformation, $\boldsymbol{i}_{1}=\left(\begin{array}{c}1_{\langle a\rangle} \\ 1_{\langle b\rangle}\end{array}\right),|\boldsymbol{i}|_{1}=\left(\begin{array}{c}\eta_{a} \\ 1-\eta_{a}\end{array}\right)$ and

$$
\Phi_{1}=\left(\begin{array}{cc}
\eta_{a} & \eta_{a} \\
\eta_{b} & 0
\end{array}\right), \quad \Phi_{2}=\left(\begin{array}{ccc}
\eta_{a a} & \eta_{a a} & 0 \\
0 & 0 & \eta_{a b} \\
\eta_{a b} & \eta_{a b} & 0
\end{array}\right)
$$

According to the property of Perron-Frobenius operator, it is well known that $P_{N}$ is contractive, the eigenvalues of $\Phi_{N}$ are less than or equal to 1 in modules. A nonnegative eigenfunction of $P$ associated with eigenvalue 1 is the density function of an invariant measure under $F$. Because $\Phi_{N}$ is nonnegative, then by the Perron-Frobenius' theorem, the maximal eigenvalue of $\Phi_{N}$ is simple, and its eigenvector can be taken that all the components are positive.

The Markov condition (1) can be expressed as $|\boldsymbol{i}|_{N}=\Phi_{N}|\boldsymbol{i}|_{N}$. This shows that $\Phi_{N}$ has an eigenvalue 1 and $|i|_{N}$ is its eigenvector. Consequently, 1 is the maximal eigenvalue of $\Phi_{N}$. Let $\boldsymbol{\rho}_{N}=\left(\rho_{w}\right)_{w \in W_{N}}$ be the eigenvector of $\Phi_{N}^{*}$ associated with eigenvalue 1 normalized in the sense $\left(\rho_{N},|i|_{N}\right)=1$. Here, $A^{*}$ denotes the transpose matrix of $A$. We call the vector $|\boldsymbol{i}|_{N}$ the interval vector of $F_{N}$, and the vector $\rho_{N}$ the density vector of $F_{N}$. Then we can express the density function of $F_{N}$-invariant measure with $\rho_{N}$.

Lemma 1. Let $R_{N}(x) \equiv\left(\boldsymbol{\rho}_{N}, \boldsymbol{i}_{N}\right)(x)=\sum_{w \in W_{N}} \rho_{w} 1_{\langle w\rangle}(x)$. Then $R_{N}(x)$ is the density function of the invariant probability measure under $F_{N}$.

PROOF. From (4)

$$
\begin{aligned}
P_{N} R_{N}(x) & =\sum_{w \in W_{N}} \rho_{w} P_{N} 1_{\langle w\rangle}(x) \\
& =\left(\Phi_{N} \boldsymbol{i}_{N}, \boldsymbol{\rho}_{N}\right)(x)
\end{aligned}
$$




$$
\begin{aligned}
& =\left(\boldsymbol{i}_{N}, \Phi_{N}^{*} \boldsymbol{\rho}_{N}\right)(x) \\
& =\left(\boldsymbol{i}_{N}, \boldsymbol{\rho}_{N}\right)(x)=R_{N}(x) .
\end{aligned}
$$

This shows that $R_{N}(x)$ is an eigenfunction of $P_{N}$ associated with eigenvalue 1 . On the other hand, from the definition of $\rho_{N}$,

$$
\begin{aligned}
\int_{[0,1]} R_{N}(x) d x & =\sum_{w \in W_{N}} \rho_{w} \int_{[0,1]} 1_{\langle w\rangle}(x) d x \\
& =\sum_{w \in W_{N}} \rho_{w}\left(|\boldsymbol{i}|_{N}\right)_{w} \\
& =\left(\boldsymbol{\rho}_{N},|\boldsymbol{i}|_{N}\right)=1 .
\end{aligned}
$$

Thus the lemma is proved.

The aim of this paper is to prove the following theorems.

THEOREM 1. The limit function $R(x)=\lim _{N \rightarrow \infty} R_{N}(x)$ exists in $L^{1}$, and $R(x)$ is the density function of the $F$-invariant probability measure.

THEOREM 2. Suppose $F$ is Bernoulli and $\xi>\frac{1}{2} \log r$, then $R(x)$ is continuous on $[0,1]$.

\section{Framework}

Before we proceed to the proof of Theorem 1, we need to examine several properties of $F_{N}$. Since the partition $\mathcal{P}_{N+1}$ is a refinement of $\mathcal{P}_{N}$, for an admissible word $w \in W_{N}$ $\langle w\rangle$ is the disjoint union of $\langle w a\rangle, w a \in W_{N+1}$. Then $1_{\langle w\rangle}(x)=\sum_{a: w a \in W_{N+1}} 1_{\langle w a\rangle}(x)$. For $w a \in W_{N}$, we get

$$
\begin{aligned}
P_{N} 1_{\langle w a\rangle}(x) & =\eta_{h(w a)} \sum_{b \in \mathcal{A}} 1_{\langle t(w a b)\rangle}(x) \\
& =\eta_{w} \sum_{b \in \mathcal{A}} 1_{\langle t(w a b)\rangle}(x) .
\end{aligned}
$$

From (3) and (5), $P_{N+1} 1_{\langle w\rangle}\left(w \in W_{N}\right)$ turns out to be

$$
\begin{aligned}
P_{N+1} 1_{\langle w\rangle}(x) & =\left(P_{N}+\left(P_{N+1}-P_{N}\right)\right) 1_{\langle w\rangle}(x) \\
& =P_{N} 1_{\langle w\rangle}(x)+\left(P_{N+1}-P_{N}\right) \sum_{a \in A} 1_{\langle w a\rangle}(x) \\
& =\eta_{w} \sum_{a \in \mathcal{A}} 1_{\langle t(w a)\rangle}(x)+\sum_{a \in A}\left(\eta_{w a}-\eta_{w}\right) \sum_{b \in A} 1_{\langle t(w a) b\rangle}(x) .
\end{aligned}
$$


Using this relation recursively, for $M<N$, and $w \in W_{M}$, we get

$$
\begin{aligned}
P_{N} 1_{\langle w\rangle}(x)= & \eta_{w} \sum_{b \in \mathcal{A}} 1_{\langle t(w) b\rangle}(x) \\
& +\sum_{k=1}^{N-M}\left(\eta_{w b_{1} b_{2} \cdots b_{k}}-\eta_{w b_{1} b_{2} \cdots b_{k-1}}\right) \sum_{b \in A} 1_{t\left(w b_{1} \cdots b_{k}\right) b}(x) .
\end{aligned}
$$

Let us rewrite this relation with a matrix, in the same way as (4).

Definition 2. For words $w, w^{\prime} \in \tilde{W}_{N}$ we say that $w^{\prime}$ is connectable to $w$ if there exists an integer $k\left(0<k<\left|w^{\prime}\right|\right)$ such that $t(w)=h^{k}\left(w^{\prime}\right)$ and the connected word $w[1] w^{\prime}$ is $F$-admissible.

Let $\tilde{\Phi}_{N}$ be a $\tilde{W}_{N} \times \tilde{W}_{N}$ matrix as

$$
\left(\tilde{\Phi}_{N}\right)_{w, w^{\prime}}= \begin{cases}\eta_{w} & t(w)=h\left(w^{\prime}\right) \\ \eta_{w[1] h\left(w^{\prime}\right)}-\eta_{w[1] h^{2}\left(w^{\prime}\right)} & \text { if }|w|<\left|w^{\prime}\right| \leq N \text { and } w^{\prime} \text { is connectable to } w \\ 0 & \text { otherwise }\end{cases}
$$

and $\tilde{\boldsymbol{i}}_{N}$ be the vector of indicator functions similarly as $\boldsymbol{i}_{N}$, that is, $\left(\tilde{\boldsymbol{i}}_{N}\right)_{w}(x)=1_{\langle w\rangle}(x)$, for $w \in \tilde{W}_{N}$. Take the example 1 , we have

$$
\tilde{\Phi}_{2}=\left(\begin{array}{ccccc}
\eta_{a} & \eta_{a} & \eta_{a a}-\eta_{a} & \eta_{a a}-\eta_{a} & \eta_{a b}-\eta_{a} \\
\eta_{b} & 0 & \eta_{b a}-\eta_{b} & \eta_{b a}-\eta_{b} & 0 \\
0 & 0 & \eta_{a a} & \eta_{a a} & 0 \\
0 & 0 & 0 & 0 & \eta_{a b} \\
0 & 0 & \eta_{a b} & \eta_{a b} & 0
\end{array}\right), \quad \tilde{\boldsymbol{i}}_{2}=\left(\begin{array}{c}
1_{\langle a\rangle} \\
1_{\langle b\rangle} \\
1_{\langle a a\rangle} \\
1_{\langle a b\rangle} \\
1_{\langle b a\rangle}
\end{array}\right)
$$

Using $\tilde{\Phi}_{N}$, the equations (6) can be written by $P_{N} 1_{\langle w\rangle}(x)=\left(\tilde{\Phi}_{N} \tilde{\boldsymbol{i}}_{N}(x)\right)_{w}$. The eigenvalues and eigenvectors of $\Phi_{1}, \Phi_{2}, \cdots, \Phi_{N}$ are related to the one of $\tilde{\Phi}_{N}$. Hence we shall be particularly interested in studying $\tilde{\Phi}_{N}$. To write this relation precisely, let us prepare the following matrices. For $k<l$, let $M_{k, l}$ be $W_{k} \times W_{l}$ matrix as

$$
\left(M_{k, l}\right)_{w w^{\prime}}= \begin{cases}1 & \text { if } w=h^{l-k}\left(w^{\prime}\right) \\ 0 & \text { otherwise }\end{cases}
$$

$M_{k, l}$ expresses the Markov structure which is naturally induced from $W_{k}$ to $W_{l}$. For $\boldsymbol{x}_{k} \in$ $\mathbf{C}^{\# W_{k}}$ and $\boldsymbol{x}_{l} \in \mathbf{C}^{\# W_{l}}$, if $\boldsymbol{x}_{k}=M_{k, l} \boldsymbol{x}_{l}$ then $\left(\boldsymbol{x}_{k}\right)_{w}=\sum_{v: h^{l-k}(v)=w}\left(\boldsymbol{x}_{l}\right)_{v}$, and if $\boldsymbol{x}_{l}=M_{k, l}^{*} \boldsymbol{x}_{k}$ then $\left(\boldsymbol{x}_{l}\right)_{w}=\left(\boldsymbol{x}_{k}\right)_{h^{l-k}(w)}$. Let us divide $\tilde{\Phi}_{N}$ into the following blocks and define $D_{i, j}$ as 
$W_{i} \times W_{j}$ matrix:

$$
\tilde{\Phi}_{N}=\left(\begin{array}{c|c} 
& D_{1, N} \\
& D_{2, N} \\
\tilde{\Phi}_{N-1} & \vdots \\
& D_{N-1, N} \\
\hline 0 & \Phi_{N}
\end{array}\right) .
$$

LEMMA 2.

$$
D_{i, j}= \begin{cases}M_{i, j-1} D_{j-1, j} & \text { if } 1 \leq i<j-1, \\ M_{j-1, j} \Phi_{j}-\Phi_{j-1} M_{j-1, j} & \text { if } i=j-1\end{cases}
$$

Proof. By the definition of $\tilde{\Phi}_{N}$, we note that $\left(D_{j-1, j}\right)_{w, w^{\prime}}=\left(D_{j-2, j}\right)_{h(w), w^{\prime}}=$ $\left(D_{i, j}\right)_{h^{j-1-i}(w), w^{\prime}}$. Then for $i<j-1$,

$$
\begin{aligned}
\left(M_{i, j-1} D_{j-1, j}\right)_{w, w^{\prime}} & =\sum_{v \in W_{j-1}}\left(M_{i, j-1}\right)_{w, v}\left(D_{j-1, j}\right)_{v, w^{\prime}} \\
& =\sum_{v: w=h^{j-1-i}(v)}\left(D_{j-1, j}\right)_{v, w^{\prime}} .
\end{aligned}
$$

If $w$ is connectable to $w^{\prime}$ then this value is equal to $\left(D_{j-1, j}\right)_{w[1] h^{2}\left(w^{\prime}\right), w^{\prime}}$. In this case $h^{j-1-i}\left(w[1] h^{2}\left(w^{\prime}\right)\right)=w$. If $w$ is not connectable to $w^{\prime}$ then it is equal to 0 . For $i=j-1$, for $w \in W_{j-1}$ and $w^{\prime} \in W_{j}$

$$
\begin{aligned}
& \left(M_{j-1, j} \Phi_{j}-\Phi_{j-1} M_{j-1, j}\right)_{w, w^{\prime}} \\
& =\sum_{v \in W_{j}}\left(M_{j-1, j}\right)_{w, v}\left(\Phi_{j}\right)_{v, w^{\prime}}-\sum_{v \in W_{j-1}}\left(\Phi_{j-1}\right)_{w, v}\left(M_{j-1, j}\right)_{v, w^{\prime}} \\
& =\sum_{v: w=h(v)}\left(\Phi_{j}\right)_{v, w^{\prime}}-\sum_{v: v=h\left(w^{\prime}\right)}\left(\Phi_{j-1}\right)_{w, v} \\
& =\sum_{v: w=h(v), t(v)=h\left(w^{\prime}\right)} \eta_{v[1] h\left(w^{\prime}\right)}-\left(\Phi_{j-1}\right)_{w, h\left(w^{\prime}\right)} \\
& = \begin{cases}\eta_{w[1] h\left(w^{\prime}\right)}-\eta_{w[1] h^{2}\left(w^{\prime}\right)} & \text { if } t(w)=h^{2}\left(w^{\prime}\right), \\
0 & \text { otherwise. }\end{cases}
\end{aligned}
$$

Therefore we get (8).

In the remainder of this section, we will consider subspaces of $\mathbf{C}^{\# \tilde{W}_{N}}$ to which $\tilde{\Phi}_{N}$ operates. Let $\delta_{w w^{\prime}}$ be

$$
\delta_{w w^{\prime}}= \begin{cases}1 & w=w^{\prime} \\ 0 & w \neq w^{\prime}\end{cases}
$$


and $\boldsymbol{e}_{w}=\left(\delta_{w w^{\prime}}\right)_{w^{\prime} \in W_{k}}\left(w \in W_{k}, k=1,2, \cdots, N\right)$. Then we easily see that

$$
\boldsymbol{e}_{w[1, k]}=M_{k+1} \boldsymbol{e}_{w[1, k+1]} \quad(k=1,2, \cdots,|w|-1) .
$$

For $w \in W_{k}$, let $\tilde{\boldsymbol{e}}_{w} \in \mathbf{C}^{\# \tilde{W}_{k}}$ be a vector

$$
\tilde{\boldsymbol{e}}_{w}=\left(\begin{array}{c}
\boldsymbol{e}_{w[1]} \\
\boldsymbol{e}_{w[1,2]} \\
\vdots \\
\boldsymbol{e}_{w[1, k-1]} \\
\boldsymbol{e}_{w}
\end{array}\right)
$$



Proposition 1. 1. The set $\left\{\tilde{\boldsymbol{e}}_{w}: w \in \tilde{W}_{N}\right\}$ forms a basis of $\mathbf{C}^{\# \tilde{W}_{N}}$.

2. Let $X_{k}$ be a linear span of $\left\{\tilde{\boldsymbol{e}}_{w}: w \in W_{k}\right\}$ then $X_{k}$ is \#列 dimensional subspace of $\mathbf{C}^{\# W_{k}}$, and $\mathbf{C}^{\# \tilde{W}_{N}}$ equals to the direct sum $X_{1} \oplus X_{2} \oplus X_{3} \oplus \cdots \oplus X_{N}$.

3. For any $\tilde{\boldsymbol{x}}_{k} \in X_{k}, k=1,2, \cdots, N$,

$$
\left(\tilde{\boldsymbol{x}}_{k}\right)_{w}=\sum_{a: w a \in \tilde{W}_{k}}\left(\tilde{\boldsymbol{x}}_{k}\right)_{w a} \quad(|w|<k) .
$$

4. $X_{k}$ is invariant under $\tilde{\Phi}_{N}$. The restriction $\left.\tilde{\Phi}_{N}\right|_{X_{k}}$ is isomorphic to $\Phi_{N}$ on $\mathbf{C}^{\# W_{N}}$.

Proof. 1. The set of vectors $\left\{\tilde{\boldsymbol{\delta}}_{w}=\left(\delta_{w w^{\prime}}\right): w \in \tilde{W}_{N}\right\}$ becomes the natural basis of $\mathbf{C}^{\# \tilde{W}_{N}}$. The claim follows from $\tilde{\boldsymbol{\delta}}_{w}=\tilde{\boldsymbol{e}}_{w}-\tilde{\boldsymbol{e}}_{h(w)}$.

2. By (10) $\operatorname{dim} X_{N}$ is at most \#列 . Moreover by the definition $\tilde{\boldsymbol{e}}_{w}\left(w \in W_{k}\right)$ are linearly independent. Thus $\operatorname{dim} X_{k}=\# W_{k}$. Take $\boldsymbol{x} \in X_{k} \cap X_{l}(k<l)$. Since $\boldsymbol{x} \in X_{k},(\boldsymbol{x})_{w}$ is equal to 0 for $|w|>k$, particularly for $|w|=l$. On the other hand $\boldsymbol{x} \in W_{l}$, this leads to the conclusion $\boldsymbol{x}=\mathbf{0}$.

3. From the definition of $\tilde{\boldsymbol{e}}_{w}$, it is obvious.

4. From the definition of $\tilde{\Phi}_{N},\left(\tilde{\Phi}_{N}\right)_{b h^{l}(w), h^{k}(w)}=\eta_{b h^{k+1}(w)}-\eta_{b h^{k+2}(w)}$, for $w \in W_{N}$ and $k<l<|w|$. Then

$$
\tilde{\Phi}_{N} \tilde{\boldsymbol{e}}_{w}=\sum_{w: b h(w) \in W_{N}} \eta_{b h(w)} \tilde{\boldsymbol{e}}_{b h(w)}
$$


here $b h(w) \in W_{N}$, therefore $\tilde{\Phi}_{N} \tilde{\boldsymbol{e}}_{w} \in X_{N}$.

Take $\boldsymbol{y}_{k} \in \mathbf{C}^{\# W_{k}}(k=1,2, \cdots, N-1)$ arbitrary, and fix them. Let $\tilde{\boldsymbol{e}}_{w}^{*}=$ $\left(\begin{array}{c}\boldsymbol{y}_{1} \\ \boldsymbol{y}_{2}-M_{1,2}^{*} \boldsymbol{y}_{1} \\ \vdots \\ \boldsymbol{e}_{w}-M_{N-1, N}^{*} \boldsymbol{y}_{N-1}\end{array}\right)$ for $w \in W_{N}$. Then $\left\{\tilde{\boldsymbol{e}}_{w}^{*}: w \in W_{N}\right\}$ is the dual basis of $\left\{\tilde{\boldsymbol{e}}_{w}: w \in W_{N}\right\}$. Indeed, by (9),

$$
\begin{aligned}
\left(\tilde{\boldsymbol{e}}_{u}, \tilde{\boldsymbol{e}}_{v}^{*}\right)= & \left(\tilde{\boldsymbol{e}}_{u[1]}, \boldsymbol{y}_{1}\right)+\sum_{i=2}^{N-1}\left\{\left(\tilde{\boldsymbol{e}}_{u[1, i]}, \boldsymbol{y}_{i}\right)-\left(M_{i-1, i}^{*} \boldsymbol{y}_{i-1}\right)\right\} \\
& +\left(\boldsymbol{e}_{u}, \boldsymbol{e}_{v}\right)-\left(M_{N-1, N}^{*} \boldsymbol{y}_{N-1}\right) \\
= & \left(\boldsymbol{y}_{1}\right)_{u[1]}+\sum_{i=2}^{N-1}\left(\boldsymbol{y}_{i}-M_{i-1, i}^{*} \boldsymbol{y}_{i-1}\right)_{u[1, i]}+\left(\boldsymbol{e}_{v}-M_{N-1, N}^{*} \boldsymbol{y}_{N-1}\right)_{u} \\
= & \left(\boldsymbol{e}_{v}\right)_{u}=\delta_{u v} .
\end{aligned}
$$

Consequently $X_{N}^{*}$, the dual space of $X_{N}$, is the linear span of $\left\{\tilde{\boldsymbol{e}}_{w}^{*}: w \in W_{N}\right\}$. In the definition of $\tilde{\boldsymbol{e}}_{w}^{*}$, we can take $\boldsymbol{y}_{1}, \boldsymbol{y}_{2} \cdots, \boldsymbol{y}_{N-1}$ arbitrarily. This means that $X_{N}^{*} \simeq \mathbf{C}^{\# \tilde{W}_{N}} / \sim_{N}$. The relation $\sim_{N}$ is defined by

$$
\tilde{\boldsymbol{x}}_{N}=\left(\begin{array}{c}
\boldsymbol{x}_{1} \\
\boldsymbol{x}_{2} \\
\vdots \\
\boldsymbol{x}_{N}
\end{array}\right) \sim_{N} \tilde{\boldsymbol{x}}_{N}^{\prime}=\left(\begin{array}{c}
\boldsymbol{x}_{1}^{\prime} \\
\boldsymbol{x}_{2}^{\prime} \\
\vdots \\
\boldsymbol{x}_{N}^{\prime}
\end{array}\right) \Leftrightarrow \sum_{k=1}^{N} M_{k, N}^{*} \boldsymbol{x}_{k}=\sum_{k=1}^{N} M_{k, N}^{*} \boldsymbol{x}_{k}^{\prime}
$$

"If we rewrite $\tilde{\boldsymbol{x}}_{N}$ and $\tilde{\boldsymbol{x}}_{N}^{\prime}$ to $\tilde{\boldsymbol{x}}_{N}=\left(\begin{array}{c}\xi_{1} \\ \xi_{2}-M_{1,2}^{*} \xi_{1} \\ \vdots \\ \xi_{N}-M_{N-1, N} \xi_{N-1}\end{array}\right)$, and $\tilde{\boldsymbol{x}}_{N}^{\prime}=$ $\left(\begin{array}{c}\xi_{1}^{\prime} \\ \xi_{2}^{\prime}-M_{1,2}^{*} \xi_{1}^{\prime} \\ \vdots \\ \xi_{N}^{\prime}-M_{N-1, N} \xi_{N-1}^{\prime}\end{array}\right)$, then this equivalent relation implies that $\xi_{N}=\xi_{N}^{\prime}$. 
Proposition 2.

$$
\tilde{\Phi}_{N}^{*}\left(\begin{array}{c}
\boldsymbol{y}_{1} \\
\boldsymbol{y}_{2}-M_{1,2}^{*} \boldsymbol{y}_{1} \\
\vdots \\
\boldsymbol{y}_{N}-M_{N-1, N}^{*} \boldsymbol{y}_{N-1}
\end{array}\right)=\left(\begin{array}{c}
\Phi_{1}^{*} \boldsymbol{y}_{1} \\
\Phi_{2}^{*} \boldsymbol{y}_{2}-M_{1,2}^{*} \Phi_{1}^{*} \boldsymbol{y}_{1} \\
\vdots \\
\Phi_{N}^{*} \boldsymbol{y}_{N}-M_{N-1, N}^{*} \Phi_{N-1}^{*} \boldsymbol{y}_{N-1}
\end{array}\right) .
$$

Especially,

$$
\tilde{\rho}_{N}=\left(\begin{array}{c}
\rho_{1} \\
\rho_{2}-M_{1,2}^{*} \rho_{1} \\
\rho_{3}-M_{2,3}^{*} \rho_{2} \\
\vdots \\
\rho_{N}-M_{N-1, N}^{*} \rho_{N-1}
\end{array}\right)
$$

is the eigenvector of $\tilde{\Phi}_{N}^{*}$ associated with eigenvalue 1 , where $\rho_{k}$ is the density vector for $F_{k}$ $(k=1,2, \cdots, N)$.

Proof. We get the proof by induction. Since $\Phi_{1}=\tilde{\Phi}_{1}$, the claim is true for $N=1$. Let

$$
\tilde{y}_{k}=\left(\begin{array}{c}
\boldsymbol{y}_{1} \\
\boldsymbol{y}_{2}-M_{1,2}^{*} \boldsymbol{y}_{1} \\
\vdots \\
\boldsymbol{y}_{k}-M_{k-1, k}^{*} \boldsymbol{y}_{k-1}
\end{array}\right) \text {, }
$$

and assume that the claim is true for $k-1$. Then for $|w|<k$, by the formula of (7), we get

$$
\left(\tilde{\Phi}_{k}^{*} \tilde{\boldsymbol{y}}_{k}\right)_{w}=\left(\tilde{\Phi}_{k-1}^{*} \tilde{\boldsymbol{y}}_{k-1}\right)_{w} .
$$

By Lemma 2,

$$
\begin{aligned}
\left(\tilde{\Phi}_{k}^{*} \tilde{\boldsymbol{y}}_{k}\right)_{w}= & \left(\left(D_{1, k}^{*} \cdots D_{k-1, k}^{*}\right) \tilde{\boldsymbol{y}}_{k-1}+\Phi_{k}^{*}\left(\boldsymbol{y}_{k}-M_{k-1, k}^{*} \boldsymbol{y}_{k-1}\right)\right)_{w} \\
= & \left(D_{1, k}^{*} \boldsymbol{y}_{1}+D_{2, k}^{*}\left(\boldsymbol{y}_{2}-M_{1,2}^{*} \boldsymbol{y}_{1}\right)+\cdots\right. \\
& +\left(D_{k-1, k}\left(\boldsymbol{y}_{k-1}-M_{k-2, k-1} \boldsymbol{y}_{k-2}\right)+\Phi_{k}^{*}\left(\boldsymbol{y}_{k}-M_{k-1, k}^{*} \boldsymbol{y}_{k-1}\right)\right)_{w} \\
= & \left(D_{k-1, k}^{*} \boldsymbol{y}_{k-1}+\Phi_{k}^{*}\left(\boldsymbol{y}_{k}-M_{k-1, k}^{*} \boldsymbol{y}_{k-1}\right)\right)_{w} \\
= & \left(\left(\Phi_{k}^{*} M_{k-1, k}^{*}-M_{k-1, k}^{*} \Phi_{k-1}^{*}\right) \boldsymbol{y}_{k-1}+\Phi_{k}^{*}\left(\boldsymbol{y}_{k}-M_{k-1, k}^{*} \boldsymbol{y}_{k-1}\right)\right)_{w} \\
= & \left(\Phi_{k}^{*} \boldsymbol{y}_{k}-M_{k-1, k}^{*} \Phi_{k-1}^{*} \boldsymbol{y}_{k-1}\right)_{w} .
\end{aligned}
$$

Hence when $|w|=k$, we get the conclusion.

In the above calculation, we get the following equation: 
LEMMA 3

$$
\left(E_{N}-\Phi_{N}^{*}\right)\left(\rho_{N}-M_{N-1, N}^{*} \rho_{N-1}\right)=D_{N-1, N}^{*} \rho_{N-1}
$$

where $E_{N}$ is the $\# W_{N}$ dimensional identity matrix.

PROOF. In (12) and (13), for $k=N$, we substitute $\boldsymbol{\rho}_{N-1}$ and $\boldsymbol{\rho}_{N}$ for $\boldsymbol{y}_{N-1}$ and $\boldsymbol{y}_{N}$, respectively. This leads to the conclusion.

By Proposition 2, to take $\boldsymbol{\rho}_{1}, \boldsymbol{\rho}_{2}, \cdots, \boldsymbol{\rho}_{N-1}$ as $\boldsymbol{y}_{1}, \boldsymbol{y}_{2}, \cdots, \boldsymbol{y}_{N-1}$, in (11) we can identify $\tilde{\Phi}_{N}$ on $X_{N}^{*}$ with $\Phi_{N}$ on $\mathbf{C}^{\# W_{N}}$.

Now, we define norms of $\boldsymbol{x}_{N}=\left(x_{w}\right)_{w \in \tilde{W}_{N}} \in X_{N}$ as follows:

$$
\left\|\boldsymbol{x}_{N}\right\|_{N} \equiv \sup _{1 \leq k \leq N} \sum_{w \in W_{k}}\left|x_{w}\right|=\sum_{w \in W_{N}}\left|x_{w}\right|
$$

Then norms of $\boldsymbol{x}_{N}^{*}=\left(x_{w}^{*}\right) \in X_{N}^{*}$ are induced by

$$
\left\|\boldsymbol{x}_{N}^{*}\right\|_{N}^{*}=\sup _{z_{N} \in X_{N},\|z\|_{N}=1}\left|\left(z_{N}, \boldsymbol{x}_{N}^{*}\right)\right|=\sup _{w \in W_{N}}\left|x_{w}^{*}\right|
$$

\section{Proof of Theorem 1}

We can now proceed to the proof of Theorem 1. This will require some additional preliminary lemmas. Let us decompose $\mathbf{C}^{\# W_{N}}$ into the generalized eigenspace of $\Phi_{N}^{*}$. Let $\lambda_{i}(i=1,2, \cdots, s)$ be the eigenvalues of $\Phi_{N}^{*}$. Since 1 is an eigenvalue of $\Phi_{N}^{*}$, we set $\lambda_{1}=1$. Set $G_{N}^{i}=\left\{\boldsymbol{x} \in \mathbf{C}^{\# W_{N}}:\left(\Phi_{N}^{*}-\lambda_{i} E_{N}\right)^{k_{i}} \boldsymbol{x}=\mathbf{0}\right\}$, then $\mathbf{C}^{\# W_{N}}=G_{N}^{1} \oplus G_{N}^{2} \oplus \cdots \oplus G_{N}^{s}$, here $k_{i}$ is the index of $\lambda_{i}$, and $E_{N}$ is the $\# W_{N}$ dimensional identity matrix. Note that $G_{N}^{1}$ is the eigenspace associated with eigenvalue 1 . Since 1 is simple, $\operatorname{dim} G_{N}^{1}=1$. Let us denote $G_{N}^{2} \oplus G_{N}^{3} \oplus \cdots \oplus G_{N}^{s}$ by $\bar{G}_{N}$, that is, $\mathbf{C}^{\# W_{N}}=G_{N}^{1} \oplus \bar{G}_{N}$.

LEMMA 4. $\rho_{N}-M_{N-1, N}^{*} \rho_{N-1}$ belongs to $\bar{G}_{N}$.

Proof. Let us decompose the vector $\boldsymbol{\rho}_{N}-M_{N-1, N}^{*} \boldsymbol{\rho}_{N-1}=x \boldsymbol{\rho}_{N}+\boldsymbol{v}$, where $\boldsymbol{v} \in \bar{G}_{N}$. Then

$$
\begin{aligned}
& \left(|\boldsymbol{i}|_{N}, \boldsymbol{\rho}_{N}-M_{N-1, N}^{*} \boldsymbol{\rho}_{N-1}\right) \\
& \quad=\left(\Phi_{N}^{j}|\boldsymbol{i}|_{N}, \boldsymbol{\rho}_{N}-M_{N-1, N}^{*} \boldsymbol{\rho}_{N-1}\right) \\
& \quad=\left(\Phi_{N}^{j}|\boldsymbol{i}|_{N}, x \boldsymbol{\rho}_{N}+\boldsymbol{v}\right)=\left(|\boldsymbol{i}|_{N},\left(\Phi_{N}^{*}\right)^{j}\left(x \boldsymbol{\rho}_{N}+\boldsymbol{v}\right)\right) \\
& \quad=\left(|\boldsymbol{i}|_{N},\left(\Phi_{N}^{*}\right)^{j} x \boldsymbol{\rho}\right)+\left(|\boldsymbol{i}|_{N},\left(\Phi_{N}^{*}\right)^{j} \boldsymbol{v}\right)=x\left(|\boldsymbol{i}|_{N}, \boldsymbol{\rho}\right)+\left(|\boldsymbol{i}|_{N},\left(\Phi_{N}^{*}\right)^{j} \boldsymbol{v}\right) .
\end{aligned}
$$


Since $\bar{G}_{N}, \Phi_{N}^{*}$ is strictly contractive on $\bar{G}_{N},\left(\Phi_{N}^{*}\right)^{j} v$ converges to $\mathbf{0}$ as $j \rightarrow \infty$. On the other hand, by the definition of $\boldsymbol{\rho}_{k},\left(\boldsymbol{\rho},|\boldsymbol{i}|_{k}\right)=1$, then

$$
\begin{aligned}
\left(|\boldsymbol{i}|_{k}, \boldsymbol{\rho}_{k}-M_{k-1, k}^{*} \boldsymbol{\rho}_{k-1}\right) & =\sum_{w a \in W_{k}}\left(\rho_{w a}-\rho_{w}\right)|\langle w a\rangle| \\
& =\sum_{w a \in W_{k}} \rho_{w a}|\langle w a\rangle|-\sum_{w \in W_{k-1}} \rho_{w} \sum_{a: w a \in W_{k}}|\langle w a\rangle| \\
& =\sum_{w a \in W_{k}} \rho_{w a}|\langle w a\rangle|-\sum_{w \in W_{k-1}} \rho_{w}|\langle w\rangle| \\
& =\left(\boldsymbol{\rho}_{k},|\boldsymbol{i}|_{k}\right)-\left(\boldsymbol{\rho}_{k-1},|\boldsymbol{i}|_{k-1}\right) \\
& =0 .
\end{aligned}
$$

Consequently, $x=0$ therefore $\rho_{N}-M_{N-1, N}^{*} \rho_{N-1}$ belongs to $\bar{G}_{N}$.

The next lemma has a crucial role in the proof of Theorem 1.

LEMma 5. For $w \in W_{\infty}$, the sequence $\left\{\left(\rho_{N}\right)_{w[1, N]}\right\}$ converges uniformly in $W_{\infty}$ as $N \rightarrow \infty$.

Proof. For simplicity, we write $\left(\boldsymbol{\rho}_{N}\right)_{w}$ instead of $\left(\boldsymbol{\rho}_{N}\right)_{w[1, N]}$. By Lemma $4, \boldsymbol{\rho}_{N}-$ $M_{N-1, N}^{*} \rho_{N-1}$ belongs to $\bar{G}_{N}$, so $E_{N}-\Phi_{N}^{*}$ is invertible on $\bar{G}_{N}$. Put $\Psi_{N}=\left.\left(E_{N}-\Phi_{N}^{*}\right)\right|_{\bar{G}_{N}} ^{-1}$. Then by Lemma 3

$$
\rho_{N}-M_{N-1, N}^{*} \rho_{N-1}=\Psi_{N} D_{N-1, N}^{*} \rho_{N-1} .
$$

Therefore,

$$
\begin{aligned}
\boldsymbol{\rho}_{N} & =\left(M_{N-1, N}^{*}+\Psi_{N} D_{N-1, N}^{*}\right) \boldsymbol{\rho}_{N-1} \\
& =\left(M_{N-1, N}^{*}+\Psi_{N} D_{N-1, N}^{*}\right)\left(M_{N-2, N-1}^{*}+\Psi_{N-1} D_{N-2, N-1}^{*}\right) \rho_{N-2} \\
& =\left(M_{N-1, N}^{*}+\Psi_{N} D_{N-1, N}^{*}\right) \cdots\left(M_{1,2}^{*}+\Psi_{2} D_{1,2}^{*}\right) \rho_{1} .
\end{aligned}
$$

On the other hand, operator norm of $D_{N, N-1}^{*}$ is evaluated as follows:

$$
\begin{aligned}
\left\|D_{N-1, N}^{*}\right\| & =\sup _{\boldsymbol{x}^{*} \in X_{N}^{*},\left\|\boldsymbol{x}^{*}\right\|_{N}=1}\left\|D_{N, N-1}^{*} \boldsymbol{x}^{*}\right\|_{N}^{*} \\
& =\sup _{w \in W_{N-1}} \sum_{b \in \mathcal{A}, b w \in W_{N}}\left|\eta_{b w}-\eta_{h(b w)}\right| \\
& \leq r \max _{w \in W_{N}}\left|\eta_{w}-\eta_{h(w)}\right| \\
& \leq r \max _{x, y \in\langle w\rangle}\left|\frac{1}{\left|F^{\prime}(x)\right|}-\frac{1}{\left|F^{\prime}(y)\right|}\right| \\
& \leq r \max _{c \in\langle w\rangle} \operatorname{Lebes}(\langle w\rangle)\left|\frac{F^{\prime \prime}(c)}{\left(F^{\prime}(c)\right)^{2}}\right|
\end{aligned}
$$


Here recall $r=\# \mathcal{A}<\infty$. Therefore from (2), for $N \geq N_{0}$, we get

$$
\left\|D_{N-1, N}^{*}\right\| \leq K_{0} e^{-(\xi-\varepsilon) N}
$$

where $K_{0}=r \cdot \max _{x \in[0,1]}\left|\frac{F^{\prime \prime}(x)}{\left(F^{\prime}(x)\right)^{2}}\right| \cdot$ On $\bar{G}_{N}, \Phi_{N}^{*}$ is strictly contractive and

$$
\left.\left(E_{N}-\Phi_{N}^{*}\right)\right|_{\bar{G}_{N}} ^{-1}=\sum_{n \geq 0}\left(\left.\Phi_{N}^{*}\right|_{\bar{G}_{N}}\right)^{n},
$$

and, since $\Psi_{N}: \bar{G}_{N} \rightarrow \bar{G}_{N}$

$$
\left\|\Psi_{N}\right\|=\left\|\left.\left(E_{N}-\Phi_{N}\right)\right|_{\bar{G}_{N}} ^{-1}\right\| \leq \frac{1}{1-\left\|\left.\Phi_{N}^{*}\right|_{\bar{G}_{N}}\right\|} .
$$

Note that the eigenvalues of $\Phi_{N}$ converge to the eigenvalues of the Perron-Frobenius operator $P$ restricted to the set of functions with bounded variation ([4]). This says that there exists $\delta>0$ such that for sufficiently large $N$

$$
\left\|\Psi_{N}\right\| \leq \frac{1}{1-\delta}<\infty
$$

Moreover, there is just one 1 on each column of $M_{N-1, N}$, so

$$
\left\|M_{N-1, N}^{*}\right\|=\sup _{\left\|x^{*}\right\|_{N}^{*}=1}\left|M_{N-1, N}^{*} x^{*}\right|=1 .
$$

Then by (15) and (2),

$$
\begin{aligned}
\left\|\rho_{N}\right\|_{N} \leq & \left\|M_{N-1, N}^{*}+\Psi_{N} D_{N-1, N}^{*}\right\|\left\|M_{N-2, N-1}^{*}+\Psi_{N-1} D_{N-2, N-1}^{*}\right\| \\
& \cdots\left\|M_{1,2}^{*}+\Psi_{2} D_{1,2}^{*}\right\|\left\|\rho_{1}\right\|_{1} \\
\leq & \left(\left\|M_{N-1, N}^{*}\right\|+\left\|\Psi_{N}\right\|\left\|D_{N-1, N}^{*}\right\|\right)\left(\left\|M_{N-2, N-1}^{*}\right\|+\left\|\Psi_{N-1}\right\|\left\|D_{N-2, N-1}^{*}\right\|\right) \\
& \cdots\left(\left\|M_{1,2}^{*}\right\|+\left\|\Psi_{2}\right\|\left\|D_{1,2}^{*}\right\|\right)\left\|\rho_{1}\right\|_{1} \\
\leq & \left(1+K_{1} \mid\left\|D_{N-1, N}^{*}\right\|\right)\left(1+K_{1}\left\|D_{N-2, N-1}^{*}\right\|\right) \cdots\left(1+K_{1}\left\|D_{1,2}^{*}\right\|\right)\left\|\rho_{1}\right\|_{1} \\
\leq & K_{2} \prod_{j=N_{0}}^{N}\left(1+K_{1} e^{-(\xi-\varepsilon) j}\right),
\end{aligned}
$$

where $K_{1}=\frac{1}{1-\delta}$, and $K_{2}=\left(\prod_{j=1}^{N_{0}-1}\left(1+K_{1}\left\|D_{j}\right\|\right)\right)\left\|\rho_{1}\right\|_{1}$. We can take $\varepsilon$ such that $0<$ $\varepsilon<\xi$, then $\sum_{j=N_{0}}^{\infty} K_{1} e^{-(\xi-\varepsilon) j}<\infty$. By the convergence of the infinite product, $\left\|\boldsymbol{\rho}_{N}\right\|_{N}$ is bounded. Then for $m>n>N_{0}$, using (14) again,

$$
\left|\left(\boldsymbol{\rho}_{m}\right)_{w}-\left(\boldsymbol{\rho}_{n}\right)_{w}\right| \leq \sum_{k=n}^{m-1}\left|\left(\left(\boldsymbol{\rho}_{k+1}\right)_{w}-\left(\boldsymbol{\rho}_{k}\right)_{w}\right)\right|
$$




$$
\begin{aligned}
& \leq \sum_{k=n}^{m-1}\left\|\boldsymbol{\rho}_{k+1}-M_{k, k+1}^{*} \boldsymbol{\rho}_{k}\right\|_{k+1} \\
& =\sum_{k=n}^{m-1}\left\|\Psi_{k+1} D_{k, k+1}^{*} \boldsymbol{\rho}_{k}\right\|_{k+1} \\
& \leq K_{3} \sum_{k=n}^{m-1} e^{-(\xi-\varepsilon) k} \\
& =K_{3} e^{-(\xi-\varepsilon) n} \sum_{k=0}^{m-n-1} e^{-(\xi-\varepsilon) k},
\end{aligned}
$$

where $K_{3}=\frac{1}{1-\delta} \sup _{N}\left\|\rho_{N}\right\|_{N}$. We can take this term arbitrarily small for large enough $m$ and $n$. So the sequence $\left\{\left(\boldsymbol{\rho}_{N}\right)_{w[1, N]}\right\}$ is a Cauchy sequence and converges uniformly on $W_{\infty}$

To prove Theorem 1, we need one more lemma which is proved in [4].

LEMMA 6. $\left\|P-P_{N}\right\| \rightarrow 0$ in $L^{1}[0,1]$.

Proof of the Theorem 1. First we will show that $\left\{R_{N}(x)\right\}_{N \in \mathbf{N}}$ is a Cauchy sequence in $L^{1}[0,1]$. By Lemma 1 , for $M>N$,

$$
\begin{aligned}
\left\|R_{M}-R_{N}\right\|_{L_{1}} & =\sum_{w \in W_{M}} \int_{\langle w\rangle}\left|\left(\rho_{M}\right)_{w}-\left(\boldsymbol{\rho}_{N}\right)_{h(w)}\right| d x \\
& =\sum_{w \in W_{M}} \operatorname{Lebes}(\langle w\rangle)\left|\left(\boldsymbol{\rho}_{M}\right)_{w}-\left(\boldsymbol{\rho}_{N}\right)_{h(w)}\right| \\
& \leq \max _{w \in W_{M}}\left|\left(\boldsymbol{\rho}_{M}\right)_{w}-\left(\boldsymbol{\rho}_{N}\right)_{h^{M-N}(w)}\right| .
\end{aligned}
$$

By Lemma 5, this converges to 0 as $M, N \rightarrow \infty$. So let $R(x)$ be the limit function of $\left\{R_{N}(x)\right\}$. Now, we show that $R(x)$ is an eigenfunction of $P$ associated with the eigenvalue 1 .

$$
\left\|P R-P_{N} R_{N}\right\| \leq\|P\|\left\|R-R_{N}\right\|+\left\|P-P_{N}\right\|\left\|R_{N}\right\| \text {. }
$$

Here by the definition of $R(x)$ and by Lemma 6, if $N$ is large enough, then we can make the right hand side of (16) arbitrarily small. Thus for any $\varepsilon>0$, we can take large enough $N$ such that

$$
\begin{aligned}
\|P R-R\| & \leq\left\|P R-P_{N} R_{N}\right\|+\left\|P_{N} R_{N}-R_{N}\right\|+\left\|R_{N}-R\right\| \\
& <\varepsilon .
\end{aligned}
$$

Therefore, $R(x)$ is an eigenfunction of $P$ associated with eigenvalue 1 . 


\section{Proof of Theorem 2 -Bernoulli case}

We will give now the proof of Theorem 2 with a direct estimation of the value $R_{N}(x)$. First we prepare the next lemma.

LEMma 7. Let $\rho_{N}$ be the density vector for $F_{N}$. For $w, w^{\prime} \in W_{N}$, if $h(w)=h\left(w^{\prime}\right)$ then $\left(\boldsymbol{\rho}_{N}\right)_{w}=\left(\boldsymbol{\rho}_{N}\right)_{w^{\prime}}$.

Proof. Denote $\left(\Phi_{N}\right)_{w}$ the $w$ 'th column of $\Phi_{N}$. From the definition of $\Phi_{N}$, if $h(w)=$ $h\left(w^{\prime}\right)$ then $\left(\Phi_{N}\right)_{w}=\left(\Phi_{N}\right)_{w^{\prime}}$. Since $\Phi_{N}^{*} \rho_{N}=\rho_{N}$,

$$
\left(\boldsymbol{\rho}_{N}\right)_{w}=\left(\left(\Phi_{N}^{*}\right)_{w}, \boldsymbol{\rho}_{N}\right)=\left(\left(\Phi_{N}^{*}\right)_{w^{\prime}}, \boldsymbol{\rho}_{N}\right)=\left(\boldsymbol{\rho}_{N}\right)_{w^{\prime}} .
$$

From now, we assume that $F$ is Bernoulli, then all the words that belong to $\mathcal{A}^{N}$ are $F$-admissible.

Now we are ready to prove Theorem 2.

Proof of THE THEOREM 2. Let $R_{N}(x)$ be the density function of the $F_{N}$-invariant probability measure. Then by Lemma $1 R_{N}(x)=\left(\rho_{N}, i_{N}\right)(x)$. Note that $R_{N}(x)$ is constant on $\langle w\rangle$ for $w \in W_{N}$. For simplicity, we denote $\left(\rho_{N}\right)_{w}=\rho_{w}$ for $w \in W_{N}$. As is well known, the Lebesgue measure is invariant with respect to $F_{1}$, so $R_{1}(x) \equiv 1$. Now we take $F_{2}$. Then for words $b_{1} b_{2}$ and $c_{1} c_{2}$, if $b_{1}=c_{1}$ then $\rho_{b_{1} b_{2}}=\rho_{c_{1} c_{2}}$ by Lemma 7 . Therefore, the points where the discontinuity of $R_{2}(x)$ might happen are restricted to the dividing points of the partition $\mathcal{P}_{1}$. Suppose $R_{2}(x)$ is not continuous at the point $x_{0}$. Since $F$ is Bernoulli, the left and the right intervals of $x_{0}$ are of the form $\left\langle a_{\nu} a_{r}\right\rangle$ and $\left\langle a_{\nu+1} a_{1}\right\rangle(v=1,2, \cdots, r-1)$, respectively. From $\rho_{2}=\Phi_{2}^{*} \rho_{2}$, for any $b, c \in \mathcal{A}$, we get

$$
\rho_{b c}=\sum_{k=1}^{r} \eta_{a_{k} b} \rho_{a_{k}} b
$$

Then,

$$
\rho_{a_{v} a_{r}}-\rho_{a_{v+1} a_{1}}=\sum_{k=1}^{r}\left(\eta_{a_{k} a_{v}}-\eta_{a_{k} a_{v+1}}\right) \rho_{a_{k} a_{v}} .
$$

Similarly, the points that $R_{N}(x)$ is not continuous are the dividing points of the partition $\mathcal{P}_{N-1}$. Therefore, for $w \in W_{N}$,

$$
\begin{aligned}
\rho_{w} & =\sum_{k=1}^{r} \eta_{a_{k} h(w)} \rho_{a_{k}} h(w) \\
& =\sum_{k=1}^{r} \eta_{a_{k} h(w)} \sum_{j=1}^{r} \eta_{a_{j} a_{k} h^{2}(w)} \rho_{a_{j} a_{k} h^{2}(w)} \\
& =\sum_{v:|v|=N-1} \rho_{v w[1]} \prod_{k=0}^{N-1} \eta_{t^{k}(v) h^{N-k}(w)} .
\end{aligned}
$$


Denote the word $a \underbrace{b b \cdots b}_{n}$ by $a b(n)$. For the partition $\mathcal{P}_{N}$, the left and the right intervals of above $x_{0}$ are of the form $\langle a_{\nu} \underbrace{a_{r} \cdots a_{r}}_{N-1}\rangle$ and $\langle a_{\nu+1} \underbrace{a_{1} \cdots a_{1}}_{N-1}\rangle$, that is, $\left\langle a_{\nu} a_{r}(N-1)\right\rangle$ and $\left\langle a_{v+1} a_{1}(N-1)\right\rangle$.

Since $\rho_{v a_{v}}=\rho_{v a_{v+1}}$, we get

$$
\begin{aligned}
& \rho_{a_{v} a_{r}(N-1)}-\rho_{a_{v+1} a_{1}(N-1)} \\
& =\sum_{v:|v|=N-1} \rho_{v a_{v}}\left(\prod_{k=0}^{N-1} \eta_{t^{k}}(v) a_{v} h^{N-k-1}\left(a_{r}(N-1)\right)\right. \\
& =\sum_{v:|v|=N-1}^{N-1} \rho_{v a_{v}}\left(\prod_{k=0}^{N-1} \eta_{t^{k}}(v) a_{v} a_{r}(k)-\prod_{k=0}^{N-1} \eta_{t^{k}}(v) a_{v+1} a_{1}(k)\right) .
\end{aligned}
$$

For fixed $v \in W_{N-1}$,

$$
\begin{aligned}
& \prod_{k=0}^{N-1} \eta_{t^{k}(v) a_{v} a_{r}(k)}-\prod_{k=0}^{N-1} \eta_{t^{k}(v) a_{v+1} a_{1}(k)} \\
& =\sum_{j=1}^{N-1}\left(\eta_{t^{j}(v) a_{v} a_{r}(j-1)}-\eta_{t^{j}(v) a_{v+1} a_{1}(j-1)}\right)\left(\prod_{k=0}^{j-1} \eta_{t^{k}}(v) a_{v} a_{r}(k) \prod_{k=j+1}^{N-1} \eta_{t^{k}}(v) a_{v+1} a_{1}(k)\right) \\
& \leq \max _{w \in W_{N}} \operatorname{Lebes}(\langle w\rangle) \max _{x \in[0,1]} \frac{\left|F^{\prime \prime}(x)\right|}{\left|F^{\prime}(x)\right|} \sum_{j=1}^{N-1}\left(\prod_{k=0}^{j-1} \eta_{t^{k}}(v) a_{v} a_{r}(k) \prod_{k=j+1}^{N-1} \eta_{t^{k}}(v) a_{v+1} a_{1}(k)\right) \\
& \leq K e^{-(\xi-\varepsilon) N}\left(\sum_{j=0}^{N_{0}-1}+\sum_{j=N_{0}}^{N-N_{0}}+\sum_{j=N-N_{0}+1}^{N-1}\right)\left(\prod_{k=0}^{j-1} \eta_{t^{k}(v) a_{v} a_{r}(k)} \prod_{k=j+1}^{N-1} \eta_{t^{k}(v) a_{v+1} a_{1}(k)}\right) .
\end{aligned}
$$

According to the note in (2), for $N>2 N_{0}$

$$
\begin{aligned}
(19) & \leq K e^{-(\xi-\varepsilon) N}\left(2 N_{0} c^{N_{0}} e^{-(\xi-\varepsilon)\left(N-N_{0}\right)}+\left(N-2 N_{0}\right) e^{-(\xi-\varepsilon) N}\right) \\
& \leq K e^{-2(\xi-\varepsilon) N}\left\{N+2 N_{0}\left(c^{N_{0}} e^{(\xi-\varepsilon) N_{0}}\right)+1\right\} .
\end{aligned}
$$

Therefore,

$$
\begin{aligned}
(18) & \leq K e^{-2(\xi-\varepsilon) N}\left(N+K^{\prime}\right) \sum_{v:|v|=N-1} \rho_{v} \\
& \leq K e^{-2(\xi-\varepsilon) N}\left(N+K^{\prime}\right) r^{N-1}\left\|\rho_{N}\right\|_{N} \\
& \leq K r^{N-1} e^{-2(\xi-\varepsilon) N}\left(N+K^{\prime}\right),
\end{aligned}
$$

where $K^{\prime}=2 N_{0}\left(c^{N_{0}} e^{\xi-\varepsilon}\right)^{N_{0}}+1$. By the assumption $\xi \geq \frac{1}{2} \log r$ and $\left\|\rho_{N}\right\|_{N}<\infty$, this converges to 0 as $N \rightarrow \infty$. 
The other discontinuities of $R_{N}(x)$ are between $\left\langle w a_{v} a_{r}(m)\right\rangle$ and $\left\langle w a_{v+1} a_{1}(m)\right\rangle$ for $w \in$ $W_{N-m-1}, m=1,2, \cdots, N-2$. All the discontinuity of $R_{N}(x)$ is of this form. Similarly to (17), we get

$$
\rho_{w a_{v} a_{1}(m)}=\sum_{v:|v|=m} \rho_{v w a_{v}} \prod_{k=0}^{m} \eta_{t^{k}(v) w a_{v} a_{r}(k)} .
$$

Therefore,

$$
\begin{aligned}
& \rho_{w a_{v} a_{r}(m)}-\rho_{w a_{v+1} a_{1}(m)} \\
& =\sum_{v:|v|=m} \rho_{v w a_{v}} \prod_{k=0}^{m} \eta_{t^{k}}(v) w a_{v} a_{r}(k)-\sum_{v:|v|=m} \rho_{v w a_{v+1}} \prod_{k=0}^{m} \eta_{t^{k}}(v) w a_{v} a_{1}(k) \\
& =\sum_{v:|v|=m} \rho_{v w a_{v}}\left(\prod_{k=0}^{m} \eta_{t^{k}(v) w a_{v} a_{r}(k)}-\prod_{k=0}^{m} \eta_{t^{k}}(v) w a_{v} a_{1}(k)\right) .
\end{aligned}
$$

For the fixed discontinuity, $m \rightarrow \infty$ as $N \rightarrow \infty$. Then by the similar calculation as (18), this difference converges to 0 . Thus the theorem is proved.

\section{References}

[ 1 ] A. LASOTA and J. YORKE, On the existence of invariant measures for piecewise monotonic transformations, Trans. Amer. Math. Soc. 186 (1973), 481-488.

[2] M. MORI, Fredholm determinant for piecewise linear transformations, Osaka J. Math. 27 (1990), 481-116.

[ 3 ] M. MoRI, Fredholm determinant for piecewise monotonic transformations, Osaka J. Math. 29 (1992), 479529.

[ 4 ] M. Mori, On the Convergence of the Spectrum of Perron-Frobenius Operators, Tokyo J. Math. 17 (1994), $1-19$.

Present Address:

Department of Natural, Sciences, Tokyo National College of Technology,

KUNUGIDA-MACHI, HACHIOJI-SHI, TOKYO 193-0997, JAPAN. 